\title{
Selección de los principales problemas de seguridad de las vacunas
}

\author{
John K. Iskander ${ }^{a}$ Jane Gidudu ${ }^{a}$ Nelson Arboleda ${ }^{a}$ Wan-Ting Huang ${ }^{b}$ \\ ${ }^{a}$ Oficina de Seguridad de la Inmunización, Oficina del Científico Principal, y bervicio de Inteligencia Epidémica, \\ Oficina de Desarrollo del Potencial de Mano de Obra y Profesional, Centros para el Control y la Prevención de \\ Enfermedades (CDC) de EE.UU., Atlanta, Ga., EE.UU.
}

\section{Palabras clave}

Inmunización · Seguridad de las vacunas · Vigilancia posterior a la aprobación

\section{Extracto}

Ejemplos recientes de los principales beneficios en salud pública de la vacunación incluyen reducciones globales de la mortalidad por sarampión y el registro de niveles bajos de enfermedades evitables por vacunas en Estados Unidos. No obstante, los problemas reales o percibidos de seguridad de las vacunas pueden ejercer un impacto adverso sobre los programas de vacunación. La monitorización sólida de la seguridad, posterior a la aprobación, que combina la vigilancia activa y la vigilancia pasiva con el uso de definiciones de casos estandarizados para los efectos adversos es la base científica para evaluar los problemas de seguridad. Entre los aspectos incipientes de la ciencia de seguridad de las vacunas destacan las redes de investigación clínica y la investigación de la comunicación del riesgo de las vacunas. Entre los problemas actuales de seguridad y de gran intensidad destacan la introducción de dos vacunas antirotavirus de segunda generación, las cuales requieren una estrecha monitorización de la invaginación intestinal. Hasta la fecha, los datos de EE.UU. no indican ningún riesgo elevado asociado a la vacuna de Merck aprobada (Rotateq ${ }^{\circledR}$ ). Una cuestión de interés global es el empleo de timerosal como agente conservante en viales de vacunas multidosis. Las revisiones independientes exhaustivas, así como la investigación publicada recientemente, han reafirmado la ausencia de asociación entre timerosal y trastornos del desarrollo neurológico, incluyendo el autismo. El uso dilatado de la vacuna antigripal anual y la planificación pandémica en el mundo desarrollado, o en vías de desarrollo, deben incluir planes para la monitorización de la seguridad. A medida que aumenta el número de vacunas recién aprobadas se están identificando cada vez más errores potencialmente evitables en la administración de vacunas e incidentes posteriores a la vacunación, como el sincope. Los médicos de atención primaria y otros que participan en la administración de vacunas deben seguir procedimientos correctos de almacenamiento, manejo y administración de las vacunas y deben participar en los sistemas de notificación de efectos adversos tras la inmunización (EATI). La Brighton Collaboration proporciona otra salida, en la cual pueden participar los médicos e investigadores interesados incrementando la base de conocimientos globales sobre seguridad de las vacunas. El aumento del conocimientos de los sistemas de seguridad de las vacunas y la participación en los mismos, a todos los niveles, de los sistemas de atención sanitaria en ámbitos tanto de países desarrollados como países en vías de desarrollo permitirá a las vacunas mantener su excelente registro de trayectoria de seguridad, dado que los datos de seguridad se utilizan para mejorar los procedimientos de inmunización.

Copyright $\odot 2008$ Nestec Ltd., Vevey/S. Karger AG, Basel

\section{Antecedentes}

\section{Beneficios globales de las vacunas}

Se considera que los programas de vacunación figuran entre las intervenciones de salud pública más beneficiosas y rentables [1-7]. En realidad, se constata que numerosos programas de vacunación establecidos están ahorrando costes cuando se consideran desde una perspectiva social $[8,9]$. Datos publicados recientemente han proporcionado ejemplos adicionales de cómo las vacunas pueden reducir la morbilidad y la

\section{KARGER}

Fax +41613061234 E-Mail karger@karger.ch www.karger.com
(C) 2008 Nestec Ltd., Vevey/S. Karger AG, Basel 0252-8185/08/0662-0093\$24.50/0

Accesible online en:

www.karger.com/ans
John Iskander, MD, MPH

Acting Director, Immunization Safety Office, Office of the Chief Science Office Centers for Disease Control and Prevention

1600 Clifton Road MS D-26, Atlanta, GA 30333 (USA)

Tel. +1 404639 8889, Fax +1 404639 8834, E-Mail jxi0@cdc.gov 
mortalidad en países tanto desarrollados como en vías de desarrollo. Roush y cols. [10] observaron niveles bajos récord o casi récord en enfermedades evitables con vacunas notificadas en Estados Unidos (EE.UU.) en 2006; además, la iniciativa de reducción de la mortalidad global por sarampión por medio de una vacuna que contenía sarampión como herramienta primordial ha superado sus objetivos fijados [11]. Los programas de vacunación pueden tener beneficios adicionales para aquellas personas no seleccionadas para la vacunación, tal como lo demuestra la reducción del estado de portador de neumococos y la subsiguiente disminución de los niveles de la enfermedad en grupos de edad no seleccionados para la vacunación [12].

\section{'Ciclo vital' de los problemas sobre seguridad de las}

\section{vacunas}

El incremento al máximo de los beneficios de las vacunas exige niveles de cobertura (captación) relativamente elevados y persistentes, de manera que se alcancen umbrales de inmunidad de rebaño y no se acumulen individuos vulnerables $[13,14]$. Paradójicamente, precisamente cuando los beneficios de las vacunas son más ostensibles y la cobertura vacunal es máxima existen más probabilidades de la aparición de problemas relacionados con la seguridad en la conciencia del público en general y los medios de comunicación [15]. Estos problemas pueden suscitarse, en parte debido a que pacientes, padres y proveedores de atención sanitaria carecen ya de experiencia de primera mano con las enfermedades evitables por vacunas. Al respecto, se ha afirmado frecuentemente que las vacunas son las víctimas de su propio éxito. Este es un patrón que ha sido observado en varios países, incluyendo Japón, Reino Unido (RU) y Nigeria, donde la pérdida de confianza del público en las vacunas fue seguida por una disminución de la cobertura, que llevó a brotes de enfermedades evitables por vacunas, a menudo con una morbilidad y una mortalidad considerables [16]. Sólo después de tales experiencias de primera mano se recobró la confianza en la vacunación y se restauró la cobertura. En este artículo se describe una estrategia proactiva para monitorizar la seguridad de las vacunas sobre una base progresiva con objeto de mantener la confianza, tanto del público como del proveedor de atención sanitaria, en la seguridad de las vacunas.

Debido al ámbito global de las vacunas y los programas de vacunación, en esta revisión comentaremos consideraciones globales con respecto a la monitorización de la seguridad de las vacunas y destacaremos varias cuestiones clave como ejemplos de casos. Una revisión completa de todos los problemas relacionados con las metodologías de seguridad de las vacunas y las cuestiones específicas sometidas a estudio escapa del alcance de este trabajo. Análogamente, aunque la monitorización de la seguridad dentro de los ensayos clínicos con vacunas es crucial, en esta revisión no se abordará detalladamente el estudio de la seguridad previo a la aprobación. Se destacarán los recursos informativos, incluyendo sitios web, que proporcionan información actualizada sobre recientes problemas incipientes relacionados con la seguridad de las vacunas.
Vigilancia de la seguridad posterior a la aprobación

La justificación fundamental para monitorizar estrechamente la seguridad de las vacunas recién aprobadas y los programas de vacunación recién instaurados es que los ensayos clínicos no tienen poder para detectar efectos adversos raros de las vacunas. Incluso los ensayos verdaderamente más extensos [17-19], en los que se incluyeron hasta 70.000 participantes, no son capaces de detectar efectos adversos vacunales más infrecuentes que 1 por cada 10.000 vacunados. Aunque los efectos secundarios raros pueden detener en ocasiones los programas de vacunación $[20,21]$, los proveedores deben entender que algunas vacunas de uso corriente han mostrado efectos secundarios raros y potencialmente graves; en estos casos, las instituciones responsables de la formulación de políticas han dictaminado que los beneficios de la vacunación para los individuos y la comunidad superan a los riesgos. Por ejemplo, las vacunas que contienen sarampión, y que han desempeñado un papel clave en la reducción de la mortalidad de esta enfermedad, se asocian a trombocitopenia (que se resuelve espontáneamente y carece de complicaciones) en aproximadamente 1 de cada 30.000 vacunados [22]. Lo mismo que con cualquier producto farmacéutico, las consideraciones de seguridad de las vacunas deben evaluarse con rigor científico; no obstante, las decisiones políticas deben tomar en consideración los beneficios y los riesgos documentados.

En algunos casos, las reacciones adversas a las vacunas pueden ser tan raras que sólo aparezcan tras una amplia utilización posterior al periodo de la aprobación. La asociación entre miopericarditis y la cepa NYC BOH de la vacuna antivariólica en aproximadamente 1 de cada 10.000 vacunados fue descubierta mucho tiempo después de lograr la erradicación global de la viruela, cuando en EE. UU. se emprendieron programas de vacunación antivariólica para militares y civiles en 2003 como parte de las actividades de preparación antibioterrorista [23]. Esto se debió en parte a la infrecuencia del fenómeno, pero también a las medidas de monitorización de la seguridad mucho más activas, emprendidas en ausencia de la enfermedad natural [21].

Otro motivo fundamental para la monitorización posterior a la aprobación es que en los ensayos, independientemente de su tamaño, se pueden excluir ciertas poblaciones [24], como personas con procesos médicos subyacentes o lactantes prematuros. Dado que las recomendaciones de vacunación, particularmente para los niños, son a menudo universales, la comunidad de salud pública tiene el deber de asegurar la seguridad de las vacunas nuevas en condiciones reales de uso y práctica diarios en diversas poblaciones de pacientes. Lo mismo que otros productos farmacéuticos aprobados, las vacunas pueden ser objeto de uso fuera de la edad recomendada por la FDA o las indicaciones de grupos de riesgo. Esto recibe el nombre de uso 'extraoficial'; la monitorización del empleo extraoficial de vacunas puede constituir un aspecto importante de la monitorización de la seguridad [25]. 


\section{Métodos}

Sistemas de monitorización de la seguridad de las vacunas

Los sistemas de notificación de efectos adversos de las vacunas, a menudo denominados sistemas de notificación espontánea (SNS, por sus siglas en inglés), constituyen una de las modalidades fundamentales por las cuales se monitoriza la seguridad de las vacunas. En este trabajo se discutirán los objetivos de tales sistemas, así como sus puntos fuertes y débiles. La notificación de reacciones adversas sospechosas de las vacunas a los SNS es uno de los medios principales en los que pueden participar los proveedores de atención sanitaria de primera línea para mejorar la seguridad de las vacunas. Todas las reacciones sospechosas se designan como efectos adversos tras la inmunización (EATI), lo que no implica que se conozca o sospeche una relación causal con la vacunación. A menudo, dichos sistemas de vigilancia pasiva son complementados por una vigilancia activa o intensificada, que no depende de la notificación de profesionales individuales y que proporciona información sobre personas y poblaciones vacunadas y no vacunadas.

Con raras excepciones, la mayoría de los informes de casos individuales de efectos adversos de vacunas no pueden atribuirse claramente a las vacunas, ni una vacuna puede ser excluida definitivamente como causa. El simple dato de la aparición de un incidente después de la vacunación no es suficiente para indicar una relación de 'causa y efecto'. Los padres y los pacientes no siempre están de acuerdo con este razonamiento, lo que realza la importancia de que los proveedores de vacunas de primera línea no sólo conozcan los aspectos básicos de la ciencia de seguridad de las vacunas sino que posean sólidas aptitudes de comunicación y empatía. Los estudios epidemiológicos que muestran riesgos elevados de incidentes en individuos vacunados en comparación con individuos no vacunados, especialmente si los datos indican un periodo de riesgo específico tras la vacunación o una asociación biológicamente plausible, proporcionan pruebas de causalidad relativamente firmes [26]. Estos estudios se realizan a menudo utilizando 'extensas bases de datos vinculadas', un modelo de vigilancia activa que combina información sobre el estado de vacunación de los pacientes, demografía y resultados médicos; US Vaccine Safety Datalink [27] es un ejemplo destacado.

Papel de las instituciones asesoras y los grupos de expertos

Uno de los motivos más importantes para emprender una monitorización completa de la seguridad de las vacunas estriba en asegurarse que las políticas y las recomendaciones de inmunización utilizan los mejores datos disponibles para fomentar el empleo de las vacunas de un modo seguro y eficaz. Los proveedores deben ser conscientes de las recomendaciones para el uso de vacunas establecidas por los grupos asesores en el país donde ejercen y seguir dichas recomendaciones. El Comité Asesor de Procedimientos de Inmunización de EE.UU. revisó recientemente las recomendaciones de inmunización generales [28], aparte de publicar y enviar on-line normas sobre el uso de vacunas en EE.UU. [29]. En conjunto, el Comité Asesor Global sobre Seguridad de las Vacunas (GACVS) de la Organización Mundial de la Salud (OMS) revisa, recopila y publica con regularidad información referente a la seguridad de vacunas tanto nuevas como ya establecidas $[6,30]$.

Con respecto a las cuestiones particularmente difíciles o problemáticas que afectan a la seguridad de las vacunas, pueden ser convocados grupos independientes para revisar exhaustivamente los datos y proporcionar sistemáticamente una orientación para las instituciones responsables de la formulación de políticas y la comunidad sanitaria y médica, así como recomendaciones para la investigación ulterior. Aunque el Instituto de Medicina (IOM) de EE.UU. [31] ha editado en el curso de las dos últimas décadas numerosos informes referentes a la seguridad de las vacunas [32], también entran dentro de esta categoría otras revisiones de datos sistemáticos como los de la Cochrane Collaboration [33]. También pueden convocarse reuniones de expertos, como la celebrada por la Oficina Nacional de Programas de Vacunación de EE.UU., en la que se revisaron las pruebas a favor de una relación causal entre la primera vacuna antirotavirus (Rotashield ${ }^{\circledR}$ ) y la invaginación intestinal [34].

\section{Definiciones estandarizadas de casos y la Brighton \\ Collaboration}

Dado que las vacunas se utilizan en el mundo entero, es esencial contar con una terminología compartida en el ámbito de la seguridad de las vacunas. La estandarización de las notificaciones de los EATI facilita la comparabilidad y la comunicación de los datos de seguridad de las vacunas, que pueden desempeñar un papel clave en el mantenimiento de la confianza en los programas de inmunización actuales.

A diferencia de la eficacia de las vacunas, la seguridad no puede medirse directamente. La seguridad sólo puede ser inferida de la ausencia relativa de efectos adversos de las vacunas. La falta de definiciones de casos estándar y de normas para los efectos adversos de las vacunas ha obstaculizado nuestra capacidad para comparar los datos de seguridad de las vacunas. La comparabilidad es importante para un conocimiento basado en pruebas científicas de la seguridad de diferentes vacunas y vacunas utilizadas en diferentes poblaciones. No obstante, antes de la institución de la Brighton Collaboration, el trabajo emprendido para elaborar definiciones de casos que pudieran utilizarse en la seguridad de la inmunización fue relativamente escaso $[35,36]$.

\section{La Brighton Collaboration}

El trabajo comenzó con la formación de una comisión de iniciativas y la creación de grupos de trabajo, compuestos de voluntarios internacionales con experiencia en seguridad de vacunas, asistencia de pacientes, productos farmacéuticos, asuntos preceptivos, salud pública y suministro de vacunas. Las normas para recoger, analizar y presentar datos de seguri- 
dad facilitan compartir y comparar los datos de vacunas de diferentes procedencias geográficas entre profesionales de seguridad de las vacunas de todo el mundo.

La Brighton Collaboration, en concordancia con la OMS y los Centros para el Control y la Prevención de Enfermedades de EE.UU. y europeos (CDC y ECDC), ha estado trabajando para desarrollar y difundir definiciones estandarizadas de casos para los EATI. Las definiciones de casos se clasifican por los niveles de pruebas disponibles, que diferirán según se hayan recogido en ensayos clínicos prospectivos o en un entorno de farmacovigilancia pasiva, o según aparezcan en un país desarrollado o un país en vías de desarrollo [37-42].

La Brighton Collaboration ha integrado a expertos voluntarios de todo el mundo para definir signos y síntomas corrientes, como fiebre [43] y erupción cutánea [44], así como entidades clínicas menos corrientes como meningitis aséptica [45] e invaginación intestinal [46]. Se dispone de una lista completa que puede ser descargada a través de un proceso de registro rápido en http://www.brightoncollaboration.org/internet/en/ index $/ \mathrm{html}$. Se han publicado definiciones completadas en la revista Vaccine y en noviembre de 2007 se había completado y publicado un total de 22 definiciones de casos.

Este trabajo constituía el primer esfuerzo estructurado para elaborar un consenso internacional sobre un 'lenguaje común' necesario para una comprensión científica e adicional de la seguridad de las vacunas. Actualmente, el empleo de las definiciones de casos de la Brighton Collaboration es recomendado por organizaciones clave en seguridad de las vacunas, entre las que destacan OMS, IOM, Food and Drug Administration (FDA) y la Agencia Europea de Medicamentos (EMEA) [32, 47-49]. Con objeto de incrementar la calidad de los informes presentados, el CAEFISS (Sistema de Vigilancia Canadiense de Efectos Adversos tras la Inmunización) ha incorporado definiciones específicas de Brighton en su estructura informativa (http://www.phac-aspc.gc.ca/im/pdf/hc4229e.pdf). La Collaboration, con sus más de 1.500 participantes profesionales voluntarios procedentes de más de 90 países, es única en cuanto a capacidad.

La elaboración de definiciones de casos globalmente aceptadas es uno de los aspectos de la estandarización de la seguridad de las vacunas. Serían precisos nuevos esfuerzos para explorar la estandarización más allá de la definición de los casos para indagar diversos aspectos de los datos, como codificación, recogida, análisis, así como diseños de estudios de seguridad de las vacunas. Aunque la discusión de estas iniciativas excede del alcance de este trabajo, los lectores interesados pueden remitirse a las siguientes referencias: [50-52].

\section{Red de datos globales propuesta sobre seguridad de las} vacunas

Históricamente, la mayoría de las vacunas fueron desarrolladas, producidas e introducidas por vez primera en países (fundamentalmente en Norteamérica y Europa) provistos de recursos considerables para evaluar la seguridad tanto en en- sayos clínicos como en el ámbito de farmacovigilancia. No obstante, la fabricación de vacunas está globalizada, con producción en Brasil, China, India y otros países. Algunas vacunas nuevas, dondequiera que sean fabricadas, se están introduciendo cada vez más predominantemente en países en vías de desarrollo, que carecen de una infraestructura extensa para monitorizar la seguridad.

El ejemplo más reciente de esta situación es la vacuna antirotavirus; una de las versiones (Rotarix ${ }^{\circledR}$, GlaxoSmithKline) fue introducida en países en vías de desarrollo antes de en EE.UU. o Europa.

Además de la Brighton Collaboration, existe otro abordaje global propuesto para evaluar la seguridad de las vacunas. En septiembre de 2007 se celebró en Annecy, Francia, una reunión de la Red de Datos Globales sobre la Seguridad de las Vacunas. Varios países desarrollados, como EE.UU., RU, y Dinamarca [53] cuentan ya con extensas bases de datos vinculadas (LLDB) para rastrear las vacunaciones y los resultados clínicos dentro de sus países. Existen bases de datos y tecnología informáticas en otros países, que permitirían el desarrollo de una Red de Datos Globales sobre Seguridad de las Vacunas. En esa reunión se dio mayor importancia al concepto de utilizar LLDB existentes para propiciar la identificación rápida de posibles relaciones causales entre efectos adversos y vacuna o vacunas asociadas. La conferencia se basaba en tres objetivos principales [Steve Black, comunicación personal]: (1) Permitir evaluaciones de problemas de seguridad en una extensa población combinada de EE.UU. y Unión Europea, que proporcionase un poder estadístico intensificado en comparación con el disponible en cualquier país individual; (2) permitir la comparación de los resultados obtenidos de un estudio en uno de los países con una población alternativa fácilmente disponible; (3) proporcionar la oportunidad para lugares en el mundo desarrollado de guiar y facilitar el desarrollo de experiencia en el mundo en vías de desarrollo. El objetivo final sería contar con una red global con representación, experiencia y tamaño suficientes para permitir abordar los problemas de seguridad de las vacunas de un modo completo, rápido y verosímil a nivel local.

Esta red alentaría la puesta en práctica estandarizada de las definiciones de casos elaboradas por Brighton utilizando datos computadorizados existentes en los países, y podría constituir la base para estudios cooperativos en varios países. La constitución de una Red de Datos Globales de Seguridad de las Vacunas ampliaría considerablemente el ámbito geográfico de la capacidad actual de seguridad de las vacunas. Permitiría verificar también los resultados obtenidos en un solo país o región en poblaciones adicionales, así como la realización de estudios en poblaciones más extensas para generar un poder estadístico adicional con objeto de identificar efectos adversos raros. Además, el desarrollo de redes de datos en lugares que actualmente carecen de ellas facilitará la evaluación local de cuestiones o hipótesis de seguridad en poblaciones de todo el mundo. Esta actitud será crítica para vacunas en fase de desarrollo (como el paludismo y la tuberculosis), que pueden ser introducidas en 
el mundo en vías de desarrollo, pero que no se utilizarán en el mundo desarrollado, y que pueden proporcionar también información valiosa sobre el riesgo potencial de efectos adversos cuyas tasas de incidencia difieren por localización, o donde las tasas endémicas de la enfermedad pueden afectar a los resultados de los análisis de riesgo y beneficio.

\section{Investigación clínica de la seguridad de las vacunas}

Aunque la aparición de efectos adversos graves tras la administración de las vacunas es rara, estos preocupan a los pacientes o a sus padres, así como a los proveedores de vacunas, que pueden afrontar la adopción de decisiones médicas complejas con respecto a vacunaciones futuras tras un EATI. En varios países, incluyendo Australia e Italia [54, 55], han puesto en práctica servicios de consultoría de inmunización clínica con objeto de proporcionar evaluación médica y orientación de expertos para pacientes que han experimentado el efecto adverso de una vacuna. A menudo, la reinmunización puede ser completada con seguridad mediante una atención y monitorización médicas estrechas [56]. En EE.UU., la Red de Evaluación Clínica de la Seguridad de la Inmunización es una red de seis centros de investigación médica, provistos de experiencia con la seguridad de la inmunización por la realización de investigaciones clínicas sobre los riesgos para la salud asociados a la inmunización. La Red de Evaluación Clínica de la Seguridad de la Inmunización participa en el diseño y la realización de investigaciones sobre efectos adversos y variación individual y trata de suministrar a los proveedores y al público normas basadas en pruebas científicas en el momento de evaluar los efectos adversos o considerar la vacunación de las personas con riesgo de presentar efectos adversos. La Red persigue el estudio de la base fisiopatológica de los EATI, así como los factores de riesgo (incluyendo los factores genéticos del hospedador) asociados al desarrollo de EATI [57]. Los protocolos de investigación actuales incluyen la evaluación del Síndrome de Guillain-Barré, asociado transitoriamente a la vacunación, el desarrollo de algoritmos y normas de reacciones de hipersensibilidad a las vacunas y la observación de niños con ciertas inmunodeficiencias que reciben vacunas de virus atenuados [58].

\section{Temas específicos de interés de la seguridad de las vacunas}

Introducción de nuevas vacunas con problemas potenciales de seguridad: El ejemplo del rotavirus

En 1999, la primera vacuna antirotavirus aprobada en EE.UU. (Rotashield) fue aclamada como un progreso en salud pública, con el potencial de disminuir la considerable morbilidad y mortalidad causadas por infecciones por rotavirus en el mundo en vías de desarrollo. No obstante, en el curso de 4 meses de la publicación de la recomendación oficial de la vacuna [59], se identificó la invaginación intestinal [46], una forma de obstrucción intestinal potencialmente grave, como problema de seguridad emanado del Sistema de Notificación de Efectos Adversos de las Vacunas (VAERS), el SNS de EE.UU. Un subsiguiente estudio controlado en el marco del Enlace de Datos de la Seguridad de las Vacunas confirmó un riesgo significativamente elevado, en términos estadísticos, de invaginación intestinal en el curso de 3 a 7 días tras la administración de la primera dosis; en octubre de 1999, fueron revocadas las recomendaciones impartidas para el producto por la Comisión Asesora sobre Procedimientos de Inmunización [60] y el fabricante retiró voluntariamente la vacuna. A pesar de la persistente controversia, el dictamen científico final de la comunidad de salud pública sobre Rotashield fue que el producto se asociaba a 1 caso adicional de invaginación intestinal por cada 10.000 receptores de la vacuna [34].

No obstante, persistía la necesidad por parte de la salud pública de disponer de vacunas antirotavirus seguras y eficaces que pudieran utilizarse globalmente. En el año 2006 se publicaron extensos ensayos con vacunas antirotavirus de generación más reciente $[18,19]$ y se aprobó en EE.UU. la vacuna Rotateq ${ }^{\circledR}$ de Merck. Hasta la fecha, ni la monitorización previa a la aprobación ni la posterior a la misma [61] mostraron una asociación causal entre el producto de Merck y la invaginación intestinal. El producto de GSK, Rotarix, fue aprobado en México y otros países y hasta la fecha ha mostrado un perfil de seguridad análogamente aceptable al de la vacuna de Merck [19]. A mediados de 2007 surgieron temores referentes a informes de enfermedad de Kawasaki tras la administración de Rotateq, previos a la aprobación de la vacuna. Los datos biológicos a favor de una asociación de esta índole son muy limitados e implican a la infección natural por rotavirus en lugar de a las cepas vacunales [62]. La Comisión Asesora Global sobre la Seguridad de las Vacunas revisó recientemente los datos disponibles sin hallar pruebas convincentes de una asociación causal entre las vacunas antirotavirus aprobadas y la enfermedad de Kawasaki [30], si bien se halla en curso un estudio activo y pasivo posterior a la aprobación.

\section{Vacunas que contienen conservantes y timerosal}

En 1999, un grupo de organismos federales de EE.UU. recomendó que los fabricantes de vacunas tomaran medidas para reducir la cantidad de timerosal (también denominado tiomersal o mertiolato), un agente conservante que contiene mercurio, incluida en vacunas infantiles rutinarias [63]. A finales de 2001 o comienzos de 2002, con la excepción de algunas formulaciones de vacuna antigripal, las vacunas infantiles utilizadas en EE.UU. carecían prácticamente de timerosal [64]. Un informe inicial del IOM había planteado la hipótesis de que el timerosal podría estar vinculado a efectos neurotóxicos. No obstante, en 2004, el IOM auspició una revisión completa del problema 'vacunas y autismo', llegando a la conclusión de que estudios bien realizados respaldaban la ausencia de toda asociación al respecto y que la investigación sobre el autismo debía desviarse en otras direcciones [65]. Este punto de 
vista ha sido sustentado por revisiones publicadas recientemente [66]. En nuevos estudios publicados [67], utilizando pruebas estandarizadas de desarrollo y un control cuidadoso de posibles factores engañosos, se sigue respaldando la ausencia de neurotoxicidad del timerosal. La cuestión del timerosal sigue bajo la monitorización de la GACVS, dado que los viales de vacunas multidosis, que contienen agentes conservantes, se utilizan todavía profusamente en todo el mundo, especialmente en entornos en los que la contaminación inadvertida de las vacunas sigue suscitando una preocupación importante.

Donde estén disponibles vacunas sin y con conservantes, las recomendaciones oficiales, como las del ACIP de EE.UU., para las vacunas antigripales [68] no expresan ninguna preferencia por el empleo de alguno de los tipos de vacuna sobre otro, debido a la ausencia de datos científicos sobre el supuesto perjuicio causado por dosis bajas de timerosal.

Los proveedores también deben conocer el trabajo preliminar realizado por Andrew Wakefield en el RU, dejando entrever un vínculo entre la vacuna triple, contra sarampión, paperas y rubéola, y el autismo [69]. Esos datos no han sido confirmados por numerosos estudios controlados y las subsiguientes revisiones de expertos [70,71]. A las luces de las inquietudes, tanto científicas como éticas, referentes al trabajo original de Wakefield, la mayoría de los autores originales de los estudios han retirado su interpretación de los datos originales [72].

\section{Seguridad de las vacunas antigripales estacionales} (anuales) y pandémicas

La gripe estacional (anual) es considerada por profesionales de la salud y el público en general como una enfermedad previsible y controlable. Debido a su aparición sistemática / estacional, su morbilidad y mortalidad significativas son a menudo subestimadas. Sujetos como ancianos y niños pequeños, así como personas con determinados procesos que afectan a la salud (como asma, diabetes o cardiopatía) presentan un riesgo elevado de complicaciones gripales graves. En EE.UU., cada año contrae la gripe del 5 al $20 \%$ de la población; más de 200.000 personas son hospitalizadas a causa de sus complicaciones y 30.000 a 50.000 , fallecen. Además, en un estudio se estimó que 250.000 a 500.000 personas mueren en todo el mundo por complicaciones gripales [73].

Es fundamental contemplar la gripe como una enfermedad global con respecto a los brotes estacionales y a la amenaza velada de gripe pandémica. Aunque el agente patógeno que causará la siguiente pandemia gripal es imprevisible, el virus H5N1 es un candidato. Desde 1997, cuando se describió el primer caso humano de infección por H5N1, los brotes han seguido siendo esporádicos y es infrecuente la transmisión de persona a persona. No obstante, su tasa de mortalidad ha sido significativa en el $60 \%$ de las infecciones humanas. Entre los preparativos para una posible pandemia causada por un virus H5N1 destaca el desarrollo de vacunas antigripales apropiadas contra H5N1; en abril de 2007, la FDA aprobó la primera vacuna contra la gripe aviaria por $\mathrm{H} 5 \mathrm{~N} 1$ para uso en humanos
[74]. Aunque el H5N1 representa la mayor amenaza actual de pandemia, la vigilancia frente a la aparición de otros virus con potencial pandémico es crítica. La triple estrategia básica para una pandemia gripal se extiende sobre la respuesta de la gripe estacional: retrasando el inicio de la pandemia, mitigando la transmisión del virus y, por último, reduciendo las tasas de transmisión para disminuir el número global de infecciones y fallecimientos resultantes.

Es probable que la vacunación en el contexto de una pandemia gripal se desarrolle durante muchos meses e implique a un gran número de personas. Una campaña de vacunación contra la gripe pandémica debe contener un plan de monitorización de la seguridad de la vacuna que se realizará a lo largo de todas las fases de la respuesta pandémica [75]. Existen varios factores relacionados con una respuesta de la vacuna de la gripe pandémica, que confiere una importancia particular a la monitorización de la seguridad de la vacuna. La escala de la respuesta requerirá la capacidad para monitorizar los considerables aumentos repentinos de la notificación de efectos adversos; la diversidad de poblaciones destinatarias de vacunación (por ejemplo, edad, sexo, procesos preexistentes que afectan a la salud, gestación) puede resultar en la aparición de efectos adversos imprevistos, raros y graves, asociados a la vacuna, y la oportunidad de la respuesta puede exigir pruebas de seguridad aceleradas previas a la aprobación, que incrementarán incluso el carácter crítico de la monitorización posterior a la aprobación. La monitorización continua de vacunas prepandémicas y pandémicas para evaluar los beneficios y los riesgos de la inmunización serán esenciales para asegurar la seguridad de la vacuna durante una pandemia. Si bien los efectos secundarios graves de la vacuna son raros, es difícil detectarlos en los ensayos clínicos que se realizan con las vacunas antes de su aprobación. CDC, junto a sus socios sanitarios y la FDA de EE.UU., desempeñará un papel crucial en asegurar la seguridad de la vacuna.

La colaboración global para monitorizar la gripe ha mejorado significativamente y se ha acelerado considerablemente en respuesta a los brotes del virus de la gripe aviaria $\mathrm{H} 5 \mathrm{~N} 1$ y la epidemia de coronavirus responsable del síndrome respiratorio agudo y grave aparecido en 2003 [73]. Se prevé que las iniciativas sanitarias globales para identificar rápidamente los brotes de enfermedades infecciosas retrasen o modifiquen, pero no prevengan, la próxima pandemia de gripe. El reto de un preparativo pandémico es mantener el interés del público, así como el interés de organizaciones privadas y públicas cruciales, en iniciativas que deben prepararse ahora para planificar una pandemia que podría no aparecer durante muchos años. En el marco de la seguridad de las vacunas, las actividades preparatorias tienen que centrarse en establecer sistemas que posean un aumento sustancial de la capacidad para monitorizar los efectos adversos de las vacunas.

Se estima que para desarrollar, analizar y fabricar $300 \mathrm{mi}-$ llones de dosis de vacuna para los EE.UU. el tiempo necesario sería de 9 a 12 meses, aunque para el país un objetivo impor- 
tante es la capacidad para producir 300 millones de series en 6 meses en el año 2011 utilizando una tecnología de base celular (que todavía no está aprobada). Durante los periodos de vacunación prepandémica y pandémica inicial se prevé que la vacuna será escasa y la demanda superará considerablemente a la oferta. Los esfuerzos de vacunación serán orientados a grupos prioritarios y la monitorización de los efectos adversos se cumplirá utilizando un abordaje multivalente para evaluar los efectos adversos graves, así como estudios específicos (subpoblaciones y poblaciones vulnerables) para determinar si un efecto adverso particular está causado por una vacuna específica. El componente más crítico de un plan de monitorización de la seguridad de las vacunas es garantizar la capacidad apropiada para monitorizar la seguridad de las vacunas en una respuesta de la gripe pandémica. Esto incluye la evaluación y el incremento de la infraestructura actual de seguridad de las vacunas para proporcionar la capacidad destinada a análisis rápidos y progresivos dentro de los parámetros de los derechos individuales de privacidad y confidencialidad [76].

Con objeto de mitigar la morbilidad y la mortalidad considerables asociadas a la gripe estacional, se han puesto en práctica abordajes completos de inmunización estacional junto a estrategias de vigilancia y tratamiento. Durante una pandemia gripal grave, sin medidas de control efectivas, se estima que la consecuencia puede ser de hasta 9 millones de hospitalizaciones y casi 2 millones de fallecimientos. La facilitación de la fabricación, la distribución, así como la administración y la monitorización de vacunas seguras y eficaces contra la gripe pandémica son componentes clave de las actividades preparatorias de la pandemia.

\section{Efectos adversos potencialmente evitables}

Tras procedimientos médicos, vacunación incluida [77], se ha observado sincope vasovagal, una pérdida transitoria de la conciencia y del tono postural debidos a un reflejo simpático anormal con recuperación espontánea. En EE.UU., la VAERS recibió y analizó informes de sincopes posteriores a la vacunación [78]; este organismo representa un sistema de vigilancia pasiva posterior a la aprobación, gestionado conjuntamente por el CDC y la FDA. Una recopilación de los datos de la VAERS durante el periodo comprendido entre 1990 y 1995, con 697 casos de sincope que aparecieron en el curso de 12 horas tras la vacunación, reveló que el 45,4\% tenía edades comprendidas entre 10 y 19 años y el 57,5\% era de sexo femenino. Con respecto a aquéllos cuyo inicio del sincope se produjo una hora tras la vacunación, el $63,2 \%$ lo sufrió en el curso de 5 minutos después de la vacunación y el 88,8\%, 15 minutos después [79].

Aunque en raras ocasiones aparece un traumatismo relacionado con el sincope posterior a la vacunación, en tal caso puede representar un riesgo vital. En una revisión que describe 107 informes de caídas inducidas por un sincope posterior a la vacunación se indica que 68 incidentes $(63,6 \%)$ resultaron con lesiones secundarias, incluyendo un caso de muerte por hemorragia intracraneal en un adolescente de 15 años de edad [80].
Para prevenir los traumatismos del sincope posterior a la vacunación, la Comisión Asesora sobre Procedimientos de Inmunización del CDC recomienda actualmente que 'los proveedores de vacunas deben considerar imperativamente la observación de los pacientes durante 15 minutos después de la vacunación. En caso de sincope, los pacientes deben ser observados hasta la resolución de los síntomas' [28]. Esta recomendación es válida para todas las edades y todas las vacunas. Se requiere investigación adicional para evaluar sistemáticamente el acatamiento del periodo de observación posterior a la vacunación y su eficacia en la prevención de traumatismos relacionados con sincopes.

Otra importante categoría de EATI evitables se refiere a errores de varios tipos en la administración de las vacunas. Estos errores fueron revisados en la literatura [81] y, ocasionalmente daños graves pueden resultar por la sustitución accidental de productos inyectables no vacunales que poseen efectos sistémicos [82]. Entre otros tipos de errores documentados destacan la vía de administración incorrecta y el uso de vacunas formuladas para adultos que se administran en niños y viceversa. Aunque en raras ocasiones los pacientes sufren algún daño, estos errores pueden suscitar también cuestiones de eficacia de las vacunas y requerir una investigación intensiva de los recursos [83]. Los proveedores deben acatar las normas de almacenamiento, manejo y administración de las vacunas $[28,30]$, asegurar su propio entrenamiento y de su personal en el uso correcto de las vacunas y podrían desear también participar en iniciativas clínicas y hospitalarias locales o más amplias, diseñadas para reducir errores médicos y mejorar la seguridad de los pacientes.

\section{Conclusiones}

Los problemas de seguridad de las vacunas revisados en este trabajo proporcionan ejemplos concretos de cómo los proveedores de vacunas de atención primaria pueden contribuir a incrementar la seguridad de las vacunas. Para vacunas recién aprobadas, como la antirotavirus, la atención a las nuevas recomendaciones, en combinación con la notificación de efectos adversos de las vacunas al SNS u otros sistemas de notificación designados, fomenta el uso óptimo de estos productos con capacidad potencial de salvar vidas. Los médicos deben mantenerse alertas sobre los problemas de seguridad de las vacunas, particularmente aquellos que han atraído una atención considerable por parte de los medios, como el caso del timerosal, y deben entender los datos científicos más actuales y ser capaces de comunicarlos a los padres interesados de un modo claro y enfático [84]. El potencial para un uso más extendido de las vacunas antigripales, independientemente de que formen o no parte de una respuesta a una pandemia real o sospechada, significa que los médicos deben ser conscientes del perfil de seguridad de las vacunas antigripales actuales [68] y colaborar con las autoridades de salud pública en los esfuerzos locales, 
regionales y nacionales de planificación en la pandemia. Por último, con objeto de reducir al mínimo el riesgo de traumatismos para pacientes de todas las edades, los médicos deben acatar las recomendaciones más actualizadas para el almacenamiento, el manejo, la administración y el seguimiento de las vacunas con objeto de prevenir errores en su administración y el sincope posterior a la vacunación.

Los proveedores de asistencia clínica implicados en cualquier aspecto del suministro de vacunas pueden participar activamente en la garantía de una vacunación segura. La ampliación de la participación de los clínicos en los sistemas de notificación de seguridad de las vacunas mejorará la vigilancia de los EATI y dará como resultado vacunas más seguras y mejora de las recomendaciones para su uso. Un pequeño número de sagaces informadores a la VAERS desencadenó la retirada de la primera vacuna antirotavirus en 1999 [85]; sin embargo, también iniciaron un proceso que ha resultado en la disponibilidad de vacunas antirotavirus nuevas y más seguras. Los proveedores deben notificar los problemas de seguridad a las autoridades locales o nacionales de salud pública a través de mecanismos establecidos, independientemente de que la vacuna implicada se haya introducido recientemente o esté ya establecida. Otra manera importante por la cual los médicos y otros expertos pueden mejorar la seguridad de las vacunas es a través de su participación en la Brighton Collaboration, mediante el trabajo con colegas internacionales para elaborar nuevas definiciones de casos o utilizar las definiciones de casos existentes en pruebas o estudios posteriores a la aprobación en los que puedan estar participando. Se dispone de información adicional en http://www.brightoncollaboration.org. Los médicos deben conocer y acceder con regularidad a fuentes fidedignas de información actual sobre las vacunas y su seguridad, como los sitios web de CDC, OMS y la Red de Seguridad de las Vacunas; los pacientes en busca de información verosímil deben remitirse también a estos sitios.

Las comunidades médica y de salud pública de todo el mundo necesitarán seguir abordando exhaustivamente los problemas de seguridad de las vacunas con objeto de mantener una cobertura vacunal elevada y cosechar todos los beneficios de salud y económicos de la vacunación. En condiciones ideales, esto implicará una respuesta científica y de comunicación coordinada. Elementos basados en datos científicos de la comunicación del riesgo de las vacunas, que los proveedores deben ser conscientes de incluir, proporcionando información exacta, manteniendo la confianza en las fuentes de información, manteniendo líneas abiertas de comunicación con los padres que ponen en duda el uso de vacunas en sus hijos y remitiendo a los pacientes a recursos fidedignos, como los sitios web [86, 87]. La estandarización de la comunicación del riesgo sobre datos científicos de la seguridad de las vacunas representará una herramienta importante para aliviar o mitigar algunos de los problemas que pueden afectar la puesta en práctica de programas de inmunización en todo el mundo.

\section{Bibliografía}

1 http://www.thecommunityguide.org/vaccine/vpd-int-prov-standing-orders.pdf.

2 World Bank: World Development Report 1993: Investing in Health. New York, Oxford University Press, 1993.

-3 Centers for Disease Control and Prevention: Ten great public health achievements - United States, 1990-1999. MMWR Morb Mortal Wkly Rep 1999;48:241-243.

4 Bloom DE, Canning D, Weston M: The value of vaccination. World Economics 2005;6:1539.

5 Centers for Disease Control and Prevention: Impact of vaccines universally recommended for children - United States, 1990-1998. MMWR Morb Mortal Wkly Rep 1999;48: 243-248.

6 Folb PI, Bernatowska E, Chen R, et al: A global perspective on vaccine safety and public health: the Global Advisory Committee on Vaccine Safety. Am J Public Health 2004;94:1926-1931.

7 Hinman AR: Public health considerations; in Plotkin SA, Mortimer EA (eds): Vaccine. Philadelphia, Saunders, 1988, pp 587-611.
8 US Office of Technology Assessment: Health Program Advisory Committee, 1981. Cost Effectiveness of Influenza Vaccination. Washington, US Government Printing Office, 1981

9 Postma MJ, Baltussen RPM, Palache AM, Wilschut JC: Further evidence for favorable cost-effectiveness of elderly influenza vaccination. Expert Rev Pharmacoeconomics Outcome Res 2006;6:215-227.

10 Roush SW, Murphy TV, Vaccine-Preventable Disease Table Working Group: Historical comparisons of morbidity and mortality for vaccine-preventable diseases in the United States. JAMA 2007;298:2155-2163.

11 Centers for Disease Control and Prevention: Progress in global measles control and mortality reduction, 2000-2006. MMWR Morb Mortal Wkly Rep 2007;56:1237-1241.

12 Poehling KA, Talbot TR, Griffin MR, et al: Invasive pneumococcal disease among infants before and after introduction of pneumococcal conjugate vaccine. JAMA 2006; 295:1668-1674.
13 Hutchins SS, Baughman AL, Orr M, et al: Vaccination levels associated with lack of measles transmission among preschool-aged populations in the United States, 1989-1991. J Infect Dis 2004;189:S108-S115.

14 Anderson RM, May RM: Infectious Diseases of Humans: Dynamics and Control. Oxford, Oxford University Press, 1992.

15 Chen RT: Vaccine risks: real, perceived and unknown. Vaccine 1999;17(suppl 3):S41S46.

16 Gangarosa EJ, Galazka AM, Wolfe CR, et al: Impact of anti-vaccine movements on pertussis control: the untold story. Lancet 1998; 351:356-361.

17 Vesikari T, Giaquinto C, Huppertz HI: Clinical trials of rotavirus vaccines in Europe. Pediatr Infect Dis J 2006;25:S42-S47.

18 Vesikari T, Matson DO, Dennehy P, et al: Rotavirus Efficacy and Safety Trial (REST) Study Team. Safety and efficacy of a pentavalent human-bovine (WC3) reassortant rotavirus vaccine. N Engl J Med 2006;354:2333. 
19 Ruiz-Palacios GM, Pérez-Schael I, Velázquez FR, et al: Human Rotavirus Vaccine Study Group. Safety and efficacy of an attenuated vaccine against severe rotavirus gastroenteritis. N Engl J Med 2006;354:11-22.

-20 Centers for Disease Control and Prevention: Suspension of rotavirus vaccine after reports of intussusception - United States, 1999. MMWR Morb Mortal Wkly Rep 2004;53: 786-789 (erratum published in MMWR Morb Mortal Wkly Rep 2004;53:879).

21 Casey CG, Iskander JK, Roper MH, et al: Adverse events associated with smallpox vaccination in the United States, January-October 2003. JAMA 2005;294:2734-2743.

-22 Rajante J, Zeller B, Treutiger I, Rosthoj S: NOPHO ITP working group and five national study groups. Vaccination associated thrombocytopenic purpura in children. Vaccine 2007;25:1838-1840.

23 Arness MK, Eckart RE, Love SS, et al: Myopericarditis following smallpox vaccination. Am J Epidemiol 2004;160:642-651.

24 Ellenberg SS, Foulkes MA, Midthun K, Goldenthal KL: Evaluating the safety of new vaccines: summary of a workshop. Am J Public Health 2005;95:800-807.

25 Izurieta HS, Haber P, Wise RP, et al: Adverse events reported following live, cold-adapted, intranasal influenza vaccine. JAMA 2005; 294:2720-2725 (erratum published in JAMA 2005;294:3092).

26 Halsey N: The science of evaluation of adverse events associated with vaccination. Semin Pediatr Infect Dis 2002;13:205-214.

-27 Destefano F, Vaccine Safety Datalink Research Group: The Vaccine Safety Datalink project. Pharmacoepidemiol Drug Saf 2001; 10:403-406.

28 Kroger AT, Atkinson WL, Marcuse EK, Pickering LK: Advisory Committee on Immunization Practices (ACIP), Centers for Disease Control and Prevention. General recommendations on immunization: recommendations of the Advisory Committee on Immunization Practices (ACIP). MMWR Recomm Rep 2006;55:1-48.

29 http://www.cdc.gov/nip/acip.

$30 \mathrm{http}: / / \mathrm{www} . w h o . i n t / v a c c i n e \_s a f e t y / e n /$.

31 http://www.iom.edu/.

32 Immunization Safety Review: Hepatitis B Vaccine and Demyelinating Neurological Disorders. Washington, Board on Health Promotion and Disease Prevention (HPDP), Institute of Medicine (IOM), 2002. http:// www.iom.edu/CMS/3793/4705/4435.aspx.

33 http://www.cochrane.org/.

- 34 Peter G, Myers MG, National Vaccine Advisory Committee, National Vaccine Program Office: Intussusception, rotavirus, and oral vaccines: summary of a workshop. Pediatrics 2002;110:e67.

35 World Health Organization: Surveillance of adverse events following immunization. Field guide for managers of immunization programmes. Global Programme for Vac- cines and Immunization. Expanded Programme on Immunisation. Geneva, World Health Organization, 1997. http://who.int/ vaccines-documents/DocsPDF/www9541. pdf.

36 Ball R, Halsey N, Braun MM, et al: Development of case definitions for acute encephalopathy, encephalitis, and multiple sclerosis reports to the Vaccine Adverse Event Reporting System. J Clin Epidemiol 2002;55: 819-824.

37 Kohl KS, Gidudu J, Bonhoeffer J, et al: The development of standardized case definitions and guidelines for adverse events following immunization. Vaccine 2007;25: 5671-5674.

38 Kohl KS, Bonhoeffer J, Braun MM, et al: The Brighton Collaboration: Creating a global standard for case definitions (and guidelines) for adverse events following immunization; in AHRQ: Advances in Patient Safety. Concepts and Methodology. Rockville, AHRQ, 2005, vol 2, pp 87-102.

39 Bonhoeffer J, Kohl K, Chen R, et al: The Brighton Collaboration-enhancing vaccine safety. Vaccine 2004;22:2046.

40 Bonhoeffer J, Heininger U, Kohl K, et al: Standardized case definitions of adverse events following immunization (AEFI). Vaccine 2004;22:547-550

41 Kohl KS, Bonhoeffer J, Chen RT, et al: The Brighton Collaboration: enhancing comparability of vaccine safety data. ISPE commentary. Pharmacoepidemiol Drug Saf 2003;12:335-340.

42 Bonhoeffer J, Kohl K, Chen R, et al: The Brighton Collaboration: addressing the need for standardized case definitions of adverse events following immunization (AEFI). Vaccine 2002;21:298-302.

43 Marcy SM, Kohl KS, Dagan R, et al: The Brighton Collaboration Fever Working Group. Fever as an adverse event following immunization: case definition and guidelines of data collection, analysis, and presentation. Vaccine 2004;22:551-556.

-44 Beigel J, Kohl KS, Khuri-Bulos N, et al: Rash including mucosal involvement: case definition and guidelines for collection, analysis, and presentation of immunization safety data. Vaccine 2007;25:5697-5706.

45 Tapiainen T, Prevots R, Izurieta HS, et al Aseptic meningitis: case definition and guidelines for collection, analysis and presentation of immunization safety data. Vaccine 2007;25:5793-5802.

46 Bines JE, Kohl KS, Forster J, et al: The Brighton Collaboration Intussusception Working Group. Acute intussusception in infants and children as an adverse event following immunization: case definition and guidelines of data collection, analysis, and presentation. Vaccine 2004;22:569-574

47 World Health Organization: WHO consultation on global monitoring of adverse events following immunization, 9-10 January 2006. Wkly Epidemiol Rec 2006;81:261-272.
48 Food and Drug Administration: Guidance for industry. Toxicity grading scale for healthy adult and adolescent volunteers enrolled in preventive vaccine clinical trials. http://www.fda.gov/cber/guidelines. htm\#toxvac.

49 European Medicines Agency: Note for guidance on the clinical evaluation of vaccines. London, EMEA, Committee for Human Medicinal Products (CHMP), 2005. http://www. emea.eu.int/pdfs/human/vwp/16465305en. pdf.

50 International Conference on Harmonisation of Technical Requirements for Registration of Pharmaceuticals for Human Use (ICH). Guidelines for clinical safety assessment (E2a-e). http://www.ich.org/ (accessed December 13, 2007)

51 Yokotsuka M: The use of a medical dictionary for regulatory activities terminology (MedDRA) in prescription-event monitoring in Japan (J-PEM). Int J Med Inform 2000; 57:139-153.

52 Moher D, Cook DJ, Eastwood S, et al: Improving the quality of reports of meta-analyses of randomised controlled trials: the QUOROM statement. Quality of Reporting of Meta-analyses. Lancet 1999;354:18961900. http://www.consort-statement.org/ Initiatives/complements.htm (accessed December 13, 2007).

53 Hviid A: Postlicensure epidemiology of childhood vaccination: the Danish experience. Expert Rev Vaccines 2006;5:641-649.

54 Marshall HS, Gold MS, Gent R, et al: Ultrasound examination of extensive limb swelling reactions after diphtheria-tetanus-acellular pertussis or reduced-antigen content diphtheria-tetanus-acellular pertussis immunization in preschool-aged children. Pediatrics 2006;118:1501-1509.

55 Zanoni G, Ferro A, Valsecchi M, Tridente G: The 'Green Channel' of the Veneto region as a model for vaccine safety monitoring in Italy. Vaccine 2005;23:2354-2358.

56 Gold M, Goodwin H, Botham S, et al: Revaccination of 421 children with a past history of an adverse vaccine reaction in a special immunisation service. Arch Dis Child 2000;83:128-131.

57 Klein NP, Fireman B, Enright A, et al: Clinical Immunization Safety Assessment Network. A role for genetics in the immune response to the varicella vaccine. Pediatr Infect Dis J 2007;26:300-305.

58 http://www.cdc.gov/od/science/iso/research_activties/cisa.htm.

59 Centers for Disease Control and Prevention: Rotavirus vaccine for the prevention of rotavirus gastroenteritis among children. MMWR Morb Mortal Wkly Rep 1999;48:120.

60 Centers for Disease Control and Prevention: Withdrawal of rotavirus vaccine recommendation. MMWR Morb Mortal Wkly Rep 1999;48:1007. 
61 Centers for Disease Control and Prevention: Postmarketing monitoring of intussusception after RotaTeq vaccination - United States, February 1, 2006-February 15, 2007. MMWR Morb Mortal Wkly Rep 2007;56: 218-222.

-62 Matsuno S, Utagawa E, Sugiura A: Association of rotavirus infection with Kawasaki syndrome. J Infect Dis 1983;148:177.

63 Thimerosal in vaccines: a joint statement of the American Academy of Pediatrics and the Public Health Service. Neonatal Netw 1999; 18:65, 72.

64 Dayan GH, Iskander J, Glasser J, et al: Tracking vaccine lot lifecycles using reports to the vaccine adverse event reporting system (VAERS). Pharmacoepidemiol Drug Saf 2005; 14:671-676.

65 Meadows M: IOM report: no link between vaccines and autism. FDA Consum 2004;38: 18-19.

66 DeStefano F: Vaccines and autism: evidence does not support a causal association. Clin Pharmacol Ther 2007;82:756-759.

-67 Thompson WW, Price C, Goodson B, et al: Vaccine Safety Datalink Team. Early thimerosal exposure and neuropsychological outcomes at 7 to 10 years. N Engl J Med 2007; 357:1281-1292.

68 Fiore AE, Shay DK, Haber P, et al: Advisory Committee on Immunization Practices (ACIP), Centers for Disease Control and Prevention (CDC). Prevention and control of influenza. Recommendations of the Advisory Committee on Immunization Practices (ACIP), 2007. MMWR Recomm Rep 2007; 56:1-54.
69 Wakefield AJ, Murch SH, Anthony A, et al: Ileal-lymphoid-nodular hyperplasia, nonspecific colitis, and pervasive developmental disorder in children. Lancet 1998;351:637641.

70 Ashraf H: US expert group rejects link between MMR and autism. Lancet 2001;357: 1341.

71 DeStefano F, Thompson WW: MMR vaccine and autism: an update of the scientific evidence. Expert Rev Vaccines 2004;3:19-22.

72 Murch SH, Anthony A, Casson DH, et al: Partial retraction. Lancet 2004;363:750.

73 Seasonal and Pandemic Influenza 2007 report: Seasonal and Pandemic Influenza Meeting. American Academy of Pediatrics (AAP), the Infectious Disease Society of America (IDSA), and the Society of Healthcare Epidemiology of America (SHEA) with participation by the Centers for Disease Control and Prevention (CDC). http:// influenza-info.org/cme/2007/monograph/ monograph.pdf.

74 Food and Drug Administration: VRBPAC briefing materials from February 27, 2007, meeting. www.fda.gov (accessed August 4 2007).

75 Iskander J, Haber P, Herrera G. Monitoring vaccine safety during an influenza pandemic. Yale J Biol Med 2005;78:265-275.

76 Centers for Disease Control and Prevention: Pandemic influenza vaccine plan, chapter 8 . December 2007. Unpublished.

77 Oster LG, Sterner U, Lindahl IL: Physiologic responses in blood phobics. Behav Res Ther 1984;22:109-117.

78 Chen RT, Rastogi SC, Mullen JR, et al: The Vaccine Adverse Event Reporting System (VAERS). Vaccine 1994;12:542-550.
79 Braun MM, Patriarca PA, Ellenberg SS: Syncope after immunization. Arch Pediatr Adolesc Med 1997;151:255-259.

80 Woo EJ, Ball R, Braun MM: Fatal syncoperelated fall after immunization. Arch Pediatr Adolesc Med 2005;159:1083.

81 Varricchio F, Reed J, VAERS Working Group: Follow-up study of medication errors reported to the vaccine adverse event reporting system (VAERS). South Med J 2006;99:486489 .

82 Varricchio F: Medication errors reported to the vaccine adverse event reporting system (VAERS). Vaccine 2002;20:3049-3051.

83 Centers for Disease Control and Prevention: Inadvertent misadministration of meningococcal conjugate vaccine - United States, June-August 2005. MMWR Morb Mortal Wkly Rep 2006;55:1016-1017.

84 Smith PJ, Kennedy AM, Wooten K, et al: Association between health care providers' influence on parents who have concerns about vaccine safety and vaccination coverage. Pediatrics 2006;118:e1287-e1292.

85 Zanardi LR, Haber P, Mootrey GT, et al: Intussusception among recipients of rotavirus vaccine: reports to the vaccine adverse event reporting system. Pediatrics 2001;107:E97.

86 Hilton S, Petticrew M, Hunt K: Parents' champions vs vested interests: who do parents believe about MMR? A qualitative study. BMC Public Health 2007;7:42.

-87 Tenrreiro KN: Time-efficient strategies to ensure vaccine risk/benefit communication. J Pediatr Nurs 2005;20:469-476. 